\title{
Average Current-Mode Control of Buck DC-DC Converter With Reduced Control Voltage Ripple
}

\author{
Dalvir K. Saini*, Alberto Reatti ${ }^{\dagger}$, and Marian K. Kazimierczuk* \\ ${ }^{*}$ Department of Electrical Engineering, Wright State University, Dayton, Ohio, USA 45435 \\ \{saini.11, marian.kazimierczuk\}@wright.edu \\ ${ }^{\dagger}$ Department of Information Engineering, University of Florence, Florence, Italy 50139 \\ albertoreatti@unifi.it
}

\begin{abstract}
This paper proposes a technique to track and regulate the "true average" current in any branch of a pulse-width modulated dc-dc power converter. An example buck converter in continuous-conduction mode is considered. A comprehensive characterization is presented for the proposed scheme. An overall dc and small-signal analysis of the inner current loop is performed. The current average current-mode control methods suffer from switching instability at low duty ratios due to a large ripple in the sensed current. Moreover, the current error amplifier's high-frequency pole located at the switching frequency neither alleviates the stability issue nor improves the dynamic response of the converter. In the proposed approach, a lowpass filter, which attenuates any high-frequency ripple is placed in the feedback path of the current loop. Consequently, the control voltage is nearly dc and proportional to the actual average value of the inductor current. The inner current loop gain transfer function of the presented circuit has been derived. The following critical path closed-loop transfer functions have been derived: reference voltage-to-inductor current and reference voltage-to-output voltage. Using practical specifications of a buck dc-dc converter, a theoretical framework to design the inner loop is presented. Verification of the theoretically predicted transfer functions and transient analysis is performed through simulations.
\end{abstract}

\section{INTRODUCTION}

Average current-mode control has been a preferred solution for current control in various applications such as LED lighting, power factor correctors, and battery chargers [1] - [7]. It is also an attractive solution in two-loop dc-dc converters. Its inherent advantages are: (a) better noise immunity, (b) absence of digital or logic circuits, and (c) no requirement for slope compensation.

The existing average current-mode control techniques have been discussed in great detail in [1] and [2]. In [1], the current error amplifier was designed for an optimum proportional gain at the point, where the down-slope of the inductor current and the up-slope of the sawtooth voltage waveform applied to the pulse-width modulator are equal. However, this optimum gain led to a high inductor current ripple; consequently, the control voltage contained a proportionally amplified ripple component, which could intersect the sawtooth voltage waveform more than once during each switching period. This resulted in switching instability imposing a limitation on both duty cycle and peak sensed current. Moreover, in [1], the crossover frequency of the uncompensated loop gain was $f_{c}=f_{s} /(2 \pi D)$, where $f_{s}$ is the switching frequency and $D$

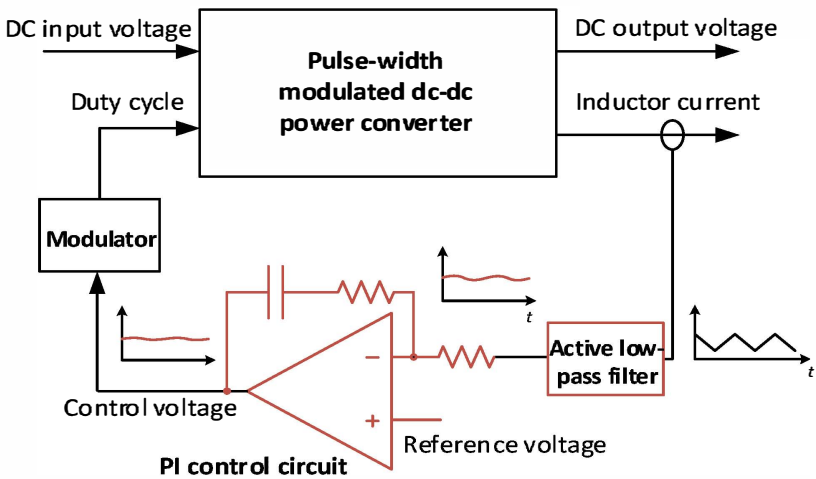

Fig. 1. Architecture of the inner loop average current-mode control technique for pulse-width modulated dc-dc power converters with a low-pass filter in the feedback path.

is the duty cycle. At low duty ratios, for example, $D \leq 0.15$, the crossover frequency $f_{c} \geq f_{s}$, which violates the Nyquist sampling theorem. Moreover, in [1] and [2], it was shown that a high-frequency pole due to the error amplifier located at $f_{s}$ eliminates the switching frequency component in the sensed current. This pole only guarantees the optimal slope condition; however, it does not contribute to an accurate average current sensing.

To overcome the above drawbacks, this paper proposes a technique, which tracks and regulates the true average current (or dc current) in any branch in the converter. A representative figure is shown in Fig. 1. A low-pass filter in the feedback path ideally eliminates the high-frequency ripple in the sensed current and allows only its average (or dc) component to the error amplifier. In the described method, the feedback voltage is ideally $\mathrm{dc}$ and is compared with the reference voltage to yield a dc control voltage. The feedback-path filter pole $f_{p}$ is located at $f_{c}<f_{p}<f_{s}$, where $f_{c}$ is the loop gain crossover frequency. The order of the stages in the loop is important. For an effective ripple suppression and true average tracking, the low-pass filter must be placed after the power stage. Therefore, in the presented scheme, the filter is placed in the feedback path. However, adding a filter pole before the power stage, e.g., in the control circuit [1], [2], [7], does not reduce the ripple. Consequently, the transistor is triggered oN at the peak inductor current instead of the average inductor current.

In summary, the major advantages of using the described 


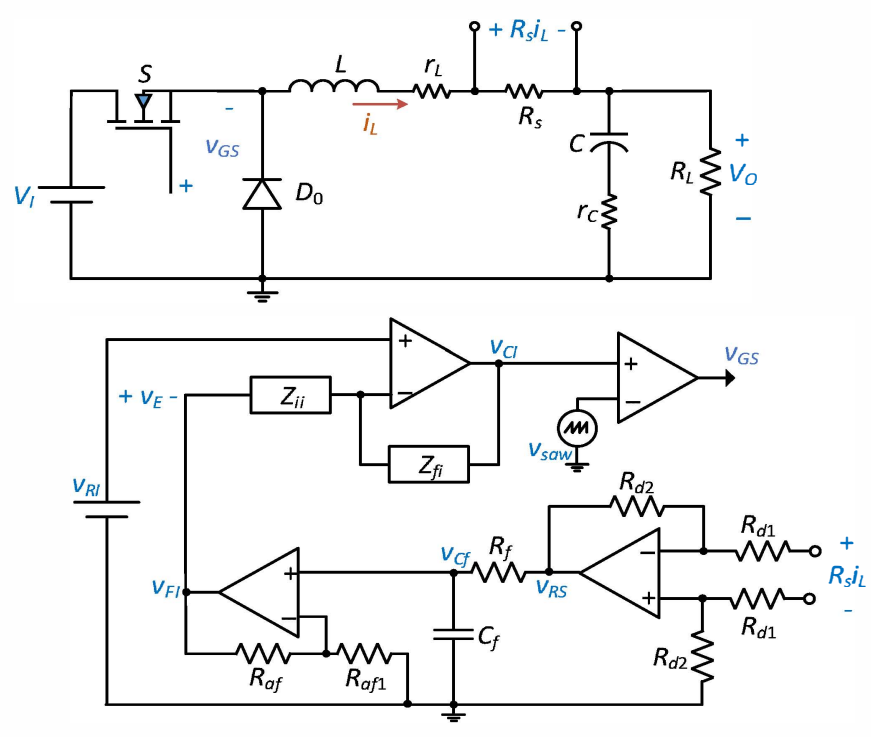

Fig. 2. Buck converter with the circuit of average current-mode control in the inner loop, including the low-pass filter.

technique are: (a) the error amplifier circuit and the low-pass filter are decoupled and both these circuits can be designed independently for improved performance, (b) switch current, diode current, or inductor current of any magnitude can be sensed, and (c) sampling is not required for the circuit description unlike in peak current-mode control.

\section{DC ANALYSIS}

\section{A. Circuit Description}

Fig. 2 shows the circuit of the average current-mode controlled buck dc-dc converter producing a reduced ripple in the control voltage. The buck converter comprises of an inductor $L$, a filter capacitor $C$, a switch $S$, a diode $D_{0}$, and a load resistor $R_{L}$. The switching frequency is $f_{s}=1 / T$ and duty cycle is $D$. The supply voltage is $V_{I}$ and the output voltage is $V_{O}$. The resistance $R_{s}$ is placed in the inductor branch to sense the inductor current. A unity gain differential amplifier is used to measure the potential difference across $R_{s}$, whose output is the sensed voltage $V_{R S}$. The voltage $V_{R S}$ is supplied to the input of a low-pass filter, designed to eliminate the switching frequency ripple component. Since the output voltage of the filter $V_{F I}$ and the reference voltage $V_{R I}$ to the inner loop must be equal, a non-inverting amplifier with a finite gain $T_{f 0}$ is used. The control voltage $V_{C I}$ at the output of the controller is compared with a sawtooth signal $v_{s a w}$, which generates the required duty cycle $D$.

\section{B. Steady-State Operation}

The inductor current $i_{L}$ comprises of the switching frequency component $\Delta i_{L}$ superimposed on the average value $I_{L}$. The potential difference across the sensed resistor $R_{s}$ is

$$
v_{R S}=R_{s} i_{L}=R_{s}\left(I_{L}+\Delta i_{L}\right) .
$$

The low-pass filter reduces or theoretically eliminates the switching frequency component of $v_{R S}$, while the non- inverting amplifier boosts its average value to that nearly equal to the reference voltage $V_{R I}$. The error voltage is given as

$$
V_{E I}=V_{R I}-V_{F I}
$$

The error amplifier such as a proportional or a proportionalintegral control circuits are used to generate the required control voltage given by

$$
V_{C I}=V_{R I}+T_{c i 0} V_{E I}
$$

where $T_{c i 0}$ is the dc gain of the control circuit. In this paper, a proportional integral control circuit is used because of large dc gain and improvement in bandwidth. The pulse-width modulator circuit is an inverting op-amp comparator. For any change in the control voltage $v_{C I}$ from $V_{C I}$ to $V_{C I}+v_{c i}$, the duty cycle $d_{T}$ also changes from $D$ to $D+d$. The control voltage-to-duty cycle transfer function of the pulsewidth modulator both for the dc and ac components is

$$
T_{m}=\frac{D}{V_{C I}}=\frac{d}{v_{c i}}=\frac{1}{V_{T m}},
$$

where $V_{T m}$ is the amplitude of the sawtooth waveform. For steady state, $V_{E I} \approx 0$ and therefore, $V_{C I} \approx V_{R I}$. From (4), the control voltage is $V_{C I}=D V_{T m}$ or $v_{c i}=V_{T m} d$.

\section{MODEL DEVELOPMENT}

A large-signal model of the average current controlled buck converter has the following sensed voltage

$$
v_{R S}=R_{s}\left(i_{L}+i_{l}\right)=R_{s}\left(I_{L}+\Delta i_{L}+i_{l}\right),
$$

where $i_{l}$ is the low-frequency, small-signal inductor current. The feedback path low-pass filter eliminates the highfrequency component $R_{s} \Delta i_{L}$. The dc component $R_{s} I_{L}$ is compared with the reference voltage set by the outer voltage loop. The low-frequency, small-signal disturbance $R_{s} i_{l}$ is attenuated by the error amplifier. Due to space constraints, only the small-signal block diagram of the closed inner current loop is shown in this paper. Fig. 3 shows the complete small-signal block diagram of the buck dc-dc converter with average current-mode control. The outer voltage loop sets the reference voltage to the inner current loop. The small-signal linear equivalent model of the buck dc-dc converter has been derived using circuit averaging technique in [6]. The smallsignal model is adopted from [5] and its duty cycle-to-inductor current transfer function $T_{p i}$, inductor current to output voltage transfer function $T_{v}$, and duty cycle-to-output voltage transfer function $T_{p}$ are presented here. Detailed derivations for $T_{p i}$, $T_{v}$, and $T_{p}$ are provided in [5].

\section{A. Power Stage Transfer Functions $T_{p i}$ and $T_{v}$}

The duty cycle-to-inductor current transfer function of the open-loop buck converter is given as

$$
T_{p i}(s)=\frac{i_{l}}{d}=T_{p i x} \frac{s+\omega_{z i}}{s^{2}+2 \xi \omega_{0} s+\omega_{0}^{2}}=T_{p i 0} \frac{1+\frac{s}{\omega_{z i}}}{1+\frac{2 \xi s}{\omega_{\bullet}}+\frac{s^{2}}{\omega_{\bullet}^{2}}},
$$




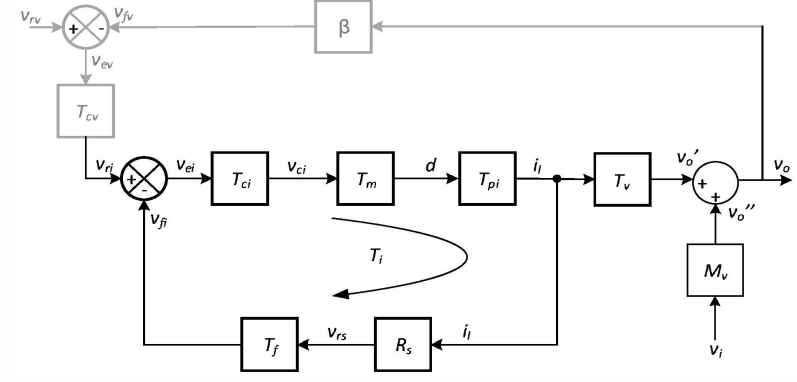

Fig. 3. Small-signal block diagram of the closed inner current loop and outer voltage loop (highlighted in gray).

where $T_{p i x}=V_{I} / L$, the gain at dc is $T_{p i 0}=V_{I} /\left(R_{L}+r\right)$, the undamped natural frequency is

$$
f_{o}=\frac{\omega_{o}}{2 \pi}=\frac{1}{2 \pi} \sqrt{\frac{R_{L}+r}{L C\left(R_{L}+r_{C}\right)}},
$$

the frequency of LHP zero is

$$
f_{z i}=\frac{\omega_{z i}}{2 \pi}=\frac{1}{2 \pi} \frac{1}{C\left(R_{L}+r_{C}\right)},
$$

and the damping coefficient is

$$
\xi=\frac{C\left(R_{L} r_{C}+r_{C} r+R_{L} r\right)+L}{2 \sqrt{L C\left(R_{L}+r_{C}\right)\left(R_{L}+r\right)}} .
$$

In the above expressions, $r_{C}$ represents the parasitic resistance of the filter capacitor and $r$ represents the equivalent averaged resistance given as $r=D r_{D S}+(1-D) R_{F}+r_{L}$, where $r_{D S}$ is the on-state resistance of the MOSFET, $R_{F}$ is the onresistance of the diode, and $r_{L}$ is the dc parasitic resistance of the inductor. The transfer function between the inductor current and the output voltage is

$$
T_{v}(s)=\frac{v_{o}}{i_{l}}=T_{v x} \frac{s+\omega_{z}}{s+\omega_{z i}}=T_{v 0} \frac{1+\frac{s}{\omega_{z}}}{1+\frac{s}{\omega_{z i}}},
$$

where $T_{v x}=R_{L} r_{C} C /\left[\left(R_{L}+r_{C}\right) C\right]$, the gain at dc is $T_{v 0}=$ $R_{L}$, and the LHP zero is $f_{z}=\omega_{z} / 2 \pi=1 /\left(2 \pi C r_{C}\right)$.

\section{B. Transfer Function of Filter and Non-Inverting Amplifier $T_{f}$}

The low-pass filter stage composed of a resistor $R_{f}$ and a capacitor $C_{f}$ is connected to the non-inverting terminal of the non-inverting amplifier. The non-inverting amplifier is composed of two resistors $R_{a f 1}$ and $R_{a f}$. The complete transfer function is

$$
T_{f}=\frac{v_{f i}}{v_{r s}}=T_{f 0}\left(\frac{\omega_{p f}}{s+\omega_{p f}}\right)=\left(1+\frac{R_{a f}}{R_{a f 1}}\right)\left(\frac{\omega_{p f}}{s+\omega_{p f}}\right),
$$

where $f_{p f}=1 /\left(2 \pi R_{f} C_{f}\right)$.

\section{Overall Transfer Function of Inner Loop $T_{k i}$}

The natural behavior of the inner uncompensated loop can be determined using the overall transfer function given as

$T_{k i}=\frac{v_{f i}}{v_{e i}}=T_{m} T_{p i} R_{s} T_{f}=T_{k i x} \frac{s+\omega_{z i}}{\left(s+\omega_{p f}\right)\left(s^{2}+2 \xi \omega_{0} s+\omega_{0}^{2}\right)}$ where $T_{m}, T_{p i}$, and $T_{f}$ are given in (4), (6), and (11), respectively. Also

$$
T_{k i x}=\frac{R_{s}}{V_{T m}}\left(1+\frac{R_{a f}}{R_{a f 1}}\right) \omega_{p f} \frac{V_{I}}{L} .
$$

From (12)

$$
T_{k i}=T_{k i 0} \frac{\left(1+\frac{s}{\omega_{z i}}\right)}{\left(1+\frac{s}{\omega_{p f}}\right)\left(1+\frac{2 \xi s}{\omega_{0}}+\frac{s^{2}}{\omega_{0}^{2}}\right)},
$$

where dc gain $T_{k i 0}$ is given by

$$
T_{k i 0}=\frac{T_{k i x} \omega_{z i}}{\omega_{f} \omega_{0}^{2}}=\frac{V_{I} R_{s}}{V_{T m}\left(R_{L}+r\right)}\left(1+\frac{R_{a f}}{R_{a f 1}}\right) .
$$

Thus, one may observe that the overall transfer function $T_{k i}$ does not depend on the filter cutoff frequency.

\section{Design EXAMPle}

\section{A. Power Stage, Low-Pass Filter, and Non-Inverting Amplifier}

A buck dc-dc converter with the following design specifications is considered to analyze the inner loop transfer functions: supply voltage is $V_{I}=28 \mathrm{~V}$, output voltage is $V_{O}=14 \mathrm{~V}$, switching frequency is $f_{s}=100 \mathrm{kHz}$, and $R_{L}=10 \Omega$. The selected MOSFET was IRF540 by International Rectifiers and the selected diode was MBR10100 by Vishay Semiconductors. From the datasheets, the on-state resistance of the MOSFET and the on-resistance of the diode were $r_{D S}=0.077 \Omega$ and $R_{F}=15 \mathrm{~m} \Omega$, respectively. The required duty cycle at an efficiency of $90 \%$ is $D=0.556$, the inductance to ensure continuous-conduction mode operation is $301 \mu \mathrm{H}$ and the capacitance is $C=68 \mu \mathrm{F}$ with its esr $r_{C}=0.111 \Omega$. The average value of the inductor current is calculated as $I_{L}=V_{O} / R_{L}=14 / 10=1.4 \mathrm{~A}$.

The amplitude of the sawtooth voltage waveform $V_{T m}=$ $5 \mathrm{~V}$. The control voltage is $V_{C I}=D V_{T m}=0.556 \times 5=$ $2.78 \mathrm{~V}$. Thus, the reference voltage $V_{R I}$ of inner loop must be set at $2.78 \mathrm{~V}$ to attain an output voltage $V_{O}=14 \mathrm{~V}$ and an inductor current $I_{L}=1.4 \mathrm{~A}$. The value of the sense resistor is chosen as $R_{s}=0.1 \Omega$. The average value of sensed voltage value $V_{R S}=R_{s} I_{L}=0.1 \times 1.4=0.14 \mathrm{~V}$. Therefore, the dc gain of the non-inverting amplifier is $T_{f 0}=V_{F I} / V_{R S} \approx$ $V_{R I} / V_{R S}=2.78 / 0.14=19.86$. Using (11) $R_{a 1}=1 \mathrm{k} \Omega$ and $R_{a f}=18.8 \mathrm{k} \Omega$. Resistance of $R_{a f}=20 \mathrm{k} \Omega$ was chosen.

The small-signal ac parameters of the open-loop power stage transfer functions are as follows. Using (7), (8), and (9), $f_{0}=$ $1.29 \mathrm{kHz}, f_{z i}=307.43 \mathrm{~Hz}$, and $\xi=0.1943$. The equivalent averaged resistance is $r=0.2578 \Omega$. For this design, the upper cutoff frequency $f_{p f}=0.5 f_{s}=50 \mathrm{kHz}$ was selected. Choosing $C_{f}=1 \mathrm{nF}$ yields

$$
R_{f}=\frac{1}{2 \pi C_{f} f_{p f}}=\frac{1}{2 \pi 1 \times 10^{-9} \times 50 \times 10^{3}}=3.18 \mathrm{k} \Omega .
$$

A standard resistor of $3.2 \mathrm{k} \Omega$ was chosen.

The transfer function $T_{k i}$ given in (12) was plotted with MATLAB for the buck converter with aforementioned specifications. Fig. 4 show the Bode magnitude and phase plots 


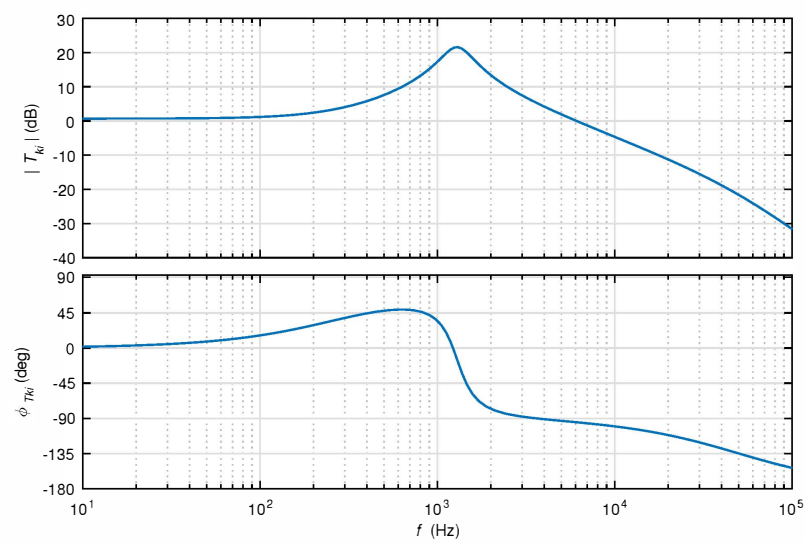

Fig. 4. Theoretically obtained magnitude and phase plots of $T_{k i}$ transfer function. (a) Magnitude of $T_{k i}$ with de gain $T_{k i 0}=1.08 \mathrm{~V} / \mathrm{V}=0.718 \mathrm{~dB}$ and $f_{c}^{\prime}=6 \mathrm{kHz}$. (b) Phase of $T_{k i}$ with $\phi_{T k i}\left(f_{c}^{\prime}\right)=-94.7^{\circ}$.

of the overall transfer function $T_{k i}$ of the buck converter with an uncompensated inner loop. The magnitude plot has a dc gain $\left|T_{k i 0}\right|=1.08 \mathrm{~V} / \mathrm{V}=0.718 \mathrm{~dB}$ and a crossover frequency $f_{c}^{\prime}=6 \mathrm{kHz}$. The phase of $T_{k i}$ at $f_{c}^{\prime}=6 \mathrm{kHz}$ is $\phi_{T k i}\left(f_{c}^{\prime}\right)=$ $-94.7^{\circ}$. The characteristics of $T_{k i}$ show a low gain at dc, a low crossover frequency, and the phase at crossover frequency must be modified to achieve a phase margin of $60^{\circ}$. The stability of the inner loop at high frequencies is ensured by the pole of the low-pass filter. The dc gain and crossover frequency must be boosted to a higher value. A classical proportional integral circuit, satisfies these requirements and its design is presented in the following section.

\section{B. Transfer Function of Control Circuit $T_{c i}$}

A proportional-integral circuit as shown in Fig. 1 has a pole at the origin that boosts the dc gain and a zero at $f_{c} / 10$ to improve the crossover frequency, where $f_{c}$ is the desired crossover frequency, and $f_{c}>f_{c}^{\prime}$. The gains of the proportional and integral parts of the control circuit are

$$
K_{p}=\frac{1}{T_{k i}\left(f_{c}\right)} \quad \text { and } \quad K_{i}=\frac{2 \pi f_{c}}{10} K_{p} .
$$

The expressions to determine component values are

$$
R_{1}=\frac{1}{K_{i} C_{1}} \quad \text { and } \quad R_{2}=K_{p} R_{1} .
$$

Thus, the transfer function $T_{c i}$ of the control circuit is

$$
T_{c i}=\frac{v_{c i}}{v_{e i}}=\frac{K_{p}\left(s+\omega_{z c i}\right)}{s}=\frac{R_{2}}{R_{1}} \frac{s+\frac{1}{R_{2} C_{1}}}{s},
$$

where $\omega_{z c i}=\frac{K_{i}}{K_{p}}=\frac{1}{R_{2} C_{1}}$. Equivalently,

$$
T_{c i}=K_{p} \omega_{z c i} \frac{1+\frac{s}{\omega_{z c i}}}{s}=T_{c i 0} \frac{1+\frac{s}{\omega_{z c i}}}{s} .
$$

The components values for the control circuit are calculated as follows. By choosing $f_{c}=10 \mathrm{kHz}$, the gain $T_{k i}$ at $f_{c}$ from the magnitude plot in Fig. 4 is $T_{k i}\left(f_{c}\right)=0.584 \mathrm{~V} / \mathrm{V}=$ $-4.68 \mathrm{~dB}$. Thus, $K_{p}=1.803, K_{i}=11.32 \times 10^{3}, C_{1}=80 \mathrm{nF}$,

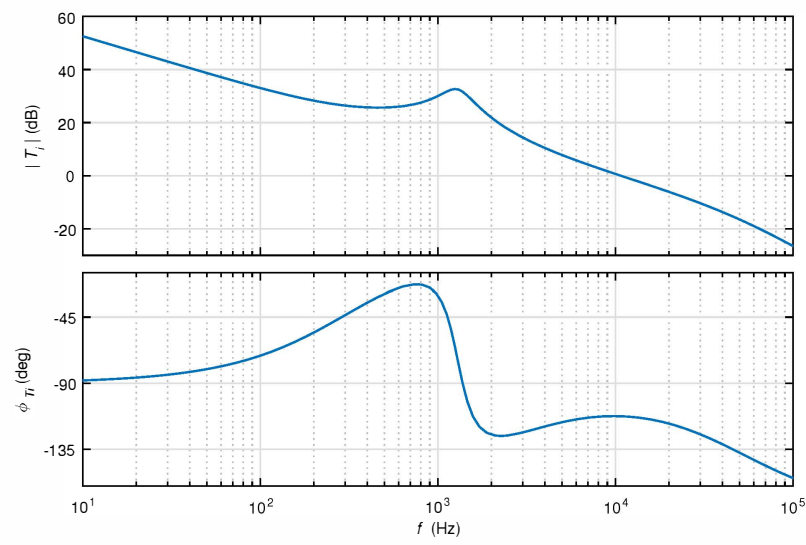

Fig. 5. Theoretically obtained magnitude and phase plots of loop gain $T_{i}$. (a) Magnitude of $T_{i}$ with dc gain $T_{i 0} \approx 562.34 \mathrm{~V} / \mathrm{V}=55 \mathrm{~dB}$ with an improved crossover frequency $f_{c}=10 \mathrm{kHz}$. (b) Phase of $T_{i}$ with $\phi_{T i}\left(f_{c}\right)=-117.5^{\circ}$ and $P M=62.5^{\circ}$.

$R_{1}=1.1 \mathrm{k} \Omega$, and $R_{2}=2 \mathrm{k} \Omega$. The zero of the control circuit is located as $f_{z c i}=1 \mathrm{kHz}$.

\section{Loop Gain of Inner Current-Loop $T_{i}$}

The loop gain of the compensated inner current loop is

$$
T_{i}=T_{k i} T_{c i}=T_{m} T_{p i} R_{s} T_{f} T_{c i},
$$

or equivalently

$$
T_{i}=T_{i x} \frac{\left(s+\omega_{z i}\right)\left(s+\omega_{z c i}\right)}{s\left(s+\omega_{p f}\right)\left(s^{2}+2 \xi \omega_{0} s+\omega_{0}^{2}\right)},
$$

where

$$
T_{i x}=T_{k i x} T_{c i x}=\frac{R_{s}}{V_{T m}}\left(1+\frac{R_{a f}}{R_{a f 1}}\right) \omega_{f} T_{p i x} \frac{R_{2}}{R_{1}} .
$$

Manipulating (22) yields

$$
T_{i}=T_{i 0} \frac{\left(1+\frac{s}{\omega_{z i}}\right)\left(1+\frac{s}{\omega_{z c i}}\right)}{s\left(1+\frac{s}{\omega_{\boldsymbol{p} f}}\right)\left(1+\frac{2 \xi s}{\omega_{\bullet}}+\frac{s^{2}}{\omega_{\bullet}^{2}}\right)},
$$

where $T_{i 0}$ is the gain at $s=0$ given by

$$
T_{i 0}=T_{k i 0} T_{c i 0}=\frac{V_{I} R_{s}}{V_{T m}\left(R_{L}+r\right)}\left(1+\frac{R_{a f}}{R_{a f 1}}\right) \frac{R_{2}}{R 1} .
$$

The magnitude and phase plots of the loop gain transfer function $T_{i}$ are shown in Fig. 5 . The dc gain is $T_{i 0} \approx 55 \mathrm{~dB}=$ $562.34 \mathrm{~V} / \mathrm{V}$, the crossover frequency is $f_{c}=10 \mathrm{kHz}$, and the phase at the new crossover frequency is $\phi_{T i}\left(f_{c}\right)=-117.5^{\circ}$. The phase margin was measured as $P M=62.5^{\circ}$ and the gain margin was $G M=\infty$. The phase of the loop gain is $-90^{\circ}$ at $\mathrm{dc}$ and is attributed to the low-frequency pole of the proportional-integral control circuit. It has been observed that the selection of the filter cutoff frequency has a significant effect on the phase margin. As the cutoff frequency $f_{p f}$ approaches the corner frequency $f_{o}$, the phase margin reduces to zero, hence, causing instability. 


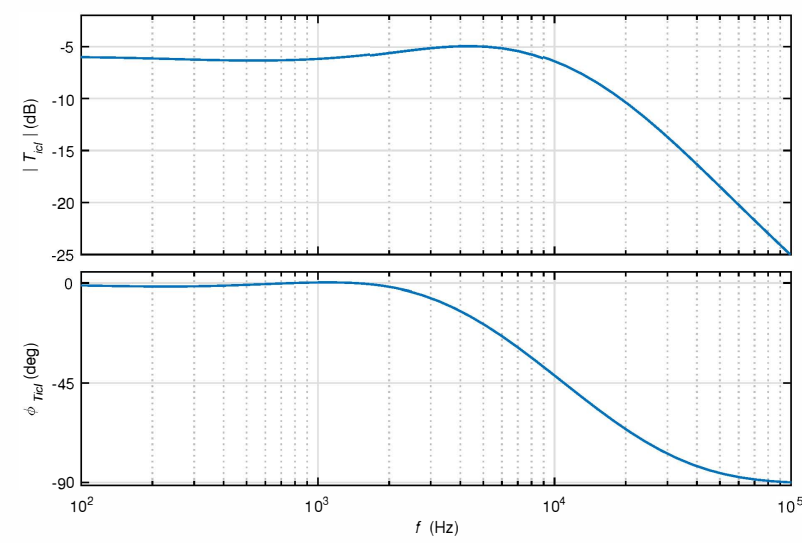

Fig. 6. Theoretically obtained magnitude and phase plots of closed-loop reference voltage-to-inductor current transfer function $T_{i c l}$. (a) $\left|T_{i c l}\right|$. (b) $\phi_{T i c l}$.
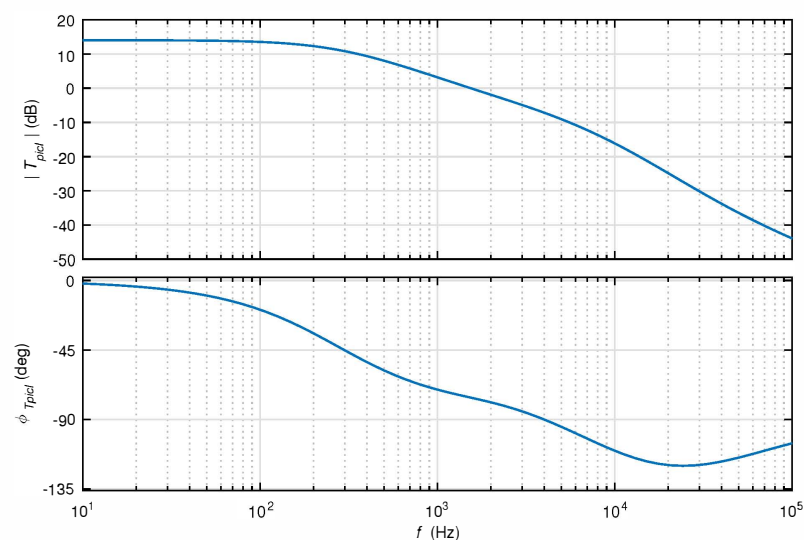

Fig. 7. Theoretically obtained magnitude and phase plots of closed-loop reference voltage-to-output voltage transfer function $T_{p i c l}$. (a) $\left|T_{p i c l}\right|$. (b) $\phi_{T \text { picl }}$.

\section{Closed-Loop Transfer Functions $T_{i c l}$ And $T_{p i c l}$}

Using the block diagram shown in Fig. 3, the closed-loop reference voltage-to-inductor current transfer function is

$$
T_{i c l}(s)=\frac{i_{l}}{v_{r i}}=\frac{T_{c i} T_{m} T_{p i}}{1+T_{i}} .
$$

Fig. 6 shows the magnitude and phase plots of the closedloop reference voltage-to-inductor current transfer function. The gain of the transfer function at dc $T_{i c l 0}$ is $-6 \mathrm{~dB}$. The closed-loop bandwidth is $B W_{c l}=17.5 \mathrm{kHz}$, while the openloop bandwidth measured through power stage duty cycleto-inductor current transfer function was $B W_{o l} \approx f_{o}=$ $1.29 \mathrm{kHz}$.

The closed-loop reference voltage-to-output voltage transfer function can be deduced as

$$
T_{p i c l}=\frac{v_{o}}{v_{r i}}=T_{i c l} T_{v}=\frac{T_{c i} T_{m} T_{p i} T_{v}}{1+T_{i}} .
$$

Fig. 7 shows the magnitude and phase plots of the closedloop reference voltage-to-output voltage transfer function. The

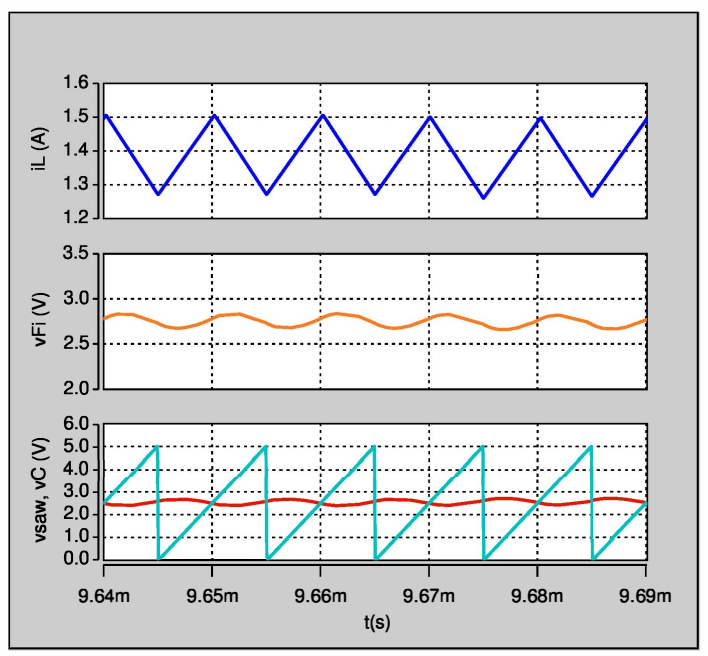

Fig. 8. Simulated steady-state waveforms of inductor current $i_{L}$, feedback voltage $v_{F I}$, control voltage $v_{C}$, and sawtooth voltage $v_{s a w}$.

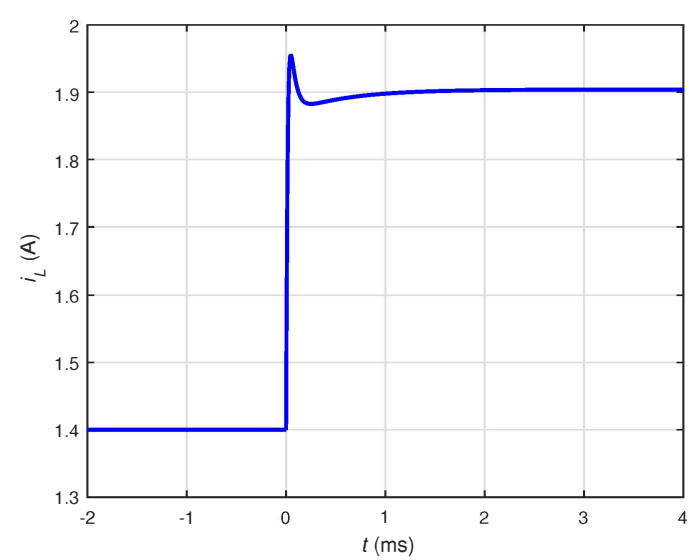

Fig. 9. Theoretically obtained plot of the inductor current $i_{L}$ for a step change in the reference voltage $v_{R I}$ by $1 \mathrm{~V}$.

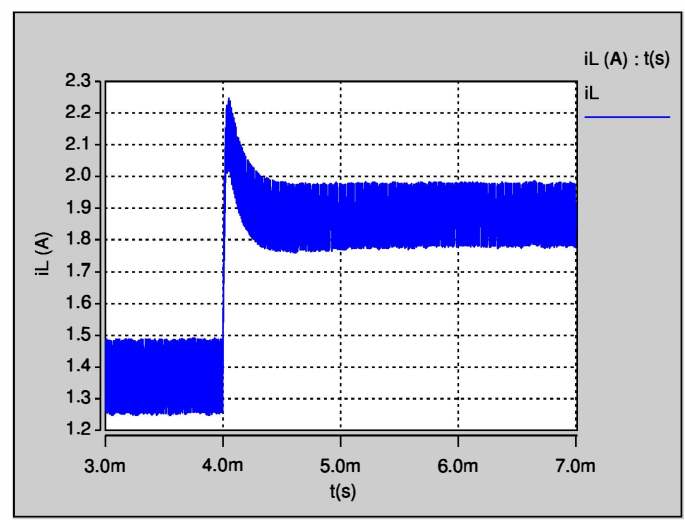

Fig. 10. SABER simulated waveform of the inductor current $i_{L}$ for a step change in the reference voltage $v_{R I}$ by $1 \mathrm{~V}$.

dc gains of $T_{i c l}$ and $T_{p i c l}$ differ by a factor equal to the load resistance. However, the overall closed-loop bandwidth of $T_{\text {picl }}$ is limited by the upper cutoff frequency of the lowpass filter network in the buck converter. 


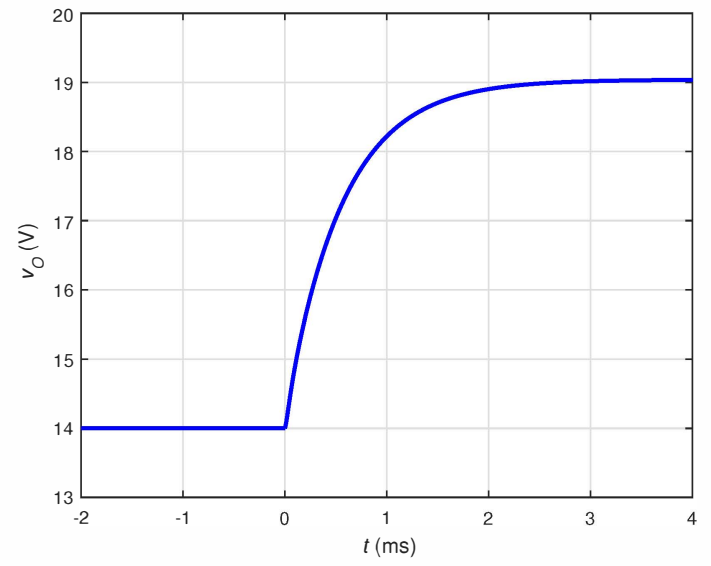

Fig. 11. Theoretically obtained plot of the output voltage $v_{O}$ for a step change in the reference voltage $v_{R I}$ by $1 \mathrm{~V}$.

\section{Vi. Model VAlidation Through Simulations}

The average current-mode controlled buck dc-dc converter in Fig. 2 was designed, built, and tested on Saber circuit simulator to verify the correctness of the theoretical predictions. The values of the components used in the simulations have been provided in Sections IV and IV-B. Fig. 8 shows the steady-state waveforms of the inductor current, feedback voltage, control voltage, and the sawtooth voltage. The feedback and control voltages are nearly dc and their values correspond to the true average value of inductor current. The ripple in the feedback voltage was reduced to $36 \%$ of its value measured in the sensed voltage.

Fig. 9 shows the theoretically obtained current response for a step change in the reference voltage $v_{R I}$ from $2.78 \mathrm{~V}$ to $3.78 \mathrm{~V}$. The plot was obtained using the transfer function provided in (26). The initial value is $1.4 \mathrm{~A}$ and the final value is $1.9 \mathrm{~A}$. The step change is $\Delta I_{L}=1.9-1.4=0.5 \mathrm{~A}$. Simulation was performed on the circuit and the resulting step response plot is as shown in Fig. 10. The average value obtained through the circuit simulation is identical to the plot obtained in Fig. 9. The dc gain obtained through the Bode plot of $T_{i c l}$ is $T_{i c l 0}=-6.2 \mathrm{~dB}=0.489$. Thus, the value $\Delta I_{L}=T_{i c l 0} \Delta V_{R I}=0.489 \times 1=0.489$ A agrees with simulation results. Similar analysis was performed to validate the transfer function $T_{p i c l}$. Fig. 11 shows the theoretically obtained voltage response for a step change in the reference voltage $v_{R I}$ from $2.78 \mathrm{~V}$ to $3.78 \mathrm{~V}$. Fig. 12 shows the step response obtained through Saber simulations. It may be observed that both theoretically obtained and simulated results are in good agreement with each other.

\section{CONCLUSION}

A true average current control technique has been proposed in this paper. This paper has developed a closed-loop analysis of buck converter operated in continuous conduction mode regulated by the average current-mode control technique. The feedback loop comprises of a low-pass filter, which is used to reduce the ripple in the control voltage. Feedback path transfer

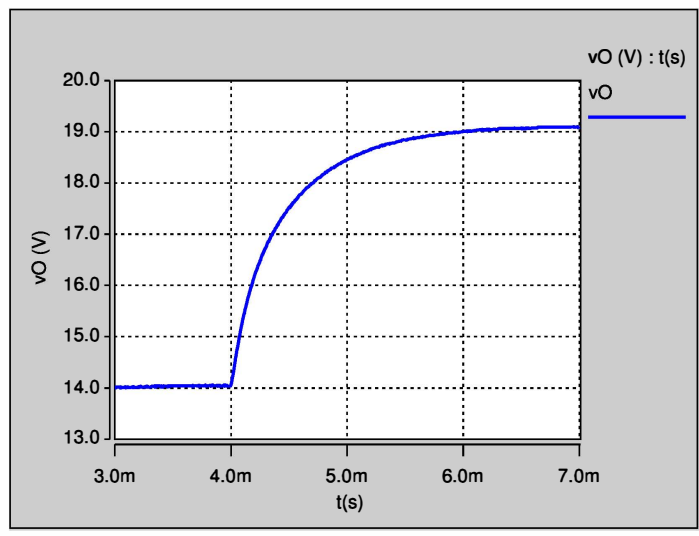

Fig. 12. SABER simulated waveform of output voltage $v_{O}$ for a step change in the reference voltage $v_{R I}$ by $1 \mathrm{~V}$.

functions have been derived. The loop gain transfer function has been established. The critical path closed-loop transfer functions such as reference voltage to the inductor current and the output voltage of the converter have been derived. The analysis in this paper have led to the following conclusions: (a) The ripple in the feedback voltage is reduced by the lowpass filter due to which the control voltage is nearly dc. Thus, problems such as switching instability or peak current sensing are avoided. These were evident drawbacks in existing average current-mode control schemes. (b) The true average component of the sensed voltage is tracked and regulated. (c) Current in any branch in the converter, irrespective of the magnitude of the ripple can be controlled. (c) The characteristics of the loop gain are unaffected by the feedback path low-pass filter up to the crossover frequency. Therefore, the control circuit can work independently to optimize the dynamic performance of the converter. (d) Noise immunity is enhanced by using the low-pass filter in the feedback path. The low-pass filter can be placed either in the feedback path (as described in this paper) or in the forward path (before the current error amplifier). However, it was observed by the authors that an ideal location for the filter was the feedback path due to better loop gain characteristics. Detailed comparison of the performance of the converter for these two cases is a topic for future study.

\section{REFERENCES}

[1] L. H. Dixon, "Average current-mode control of switching power supplies," Unitrode Power Supply Design Seminar Manual, 1990.

[2] J. Sun and R. M. Bass, "Modeling and practical design issues for average current control," in Proc. Applied Power Electron. Conf. and Expo., Mar. 1999, Dallas, TX, vol. 2, pp. 980-986.

[3] R. D. Middlebrook, "Topics in multiple-loop regulators and current-mode programming," IEEE Trans. Power Electron., vol. PE-2, no. 2, pp. 109125, Apr. 1987.

[4] N. Kondrath and M. K. Kazimierczuk, "Comparison of Wide- and HighFrequency Duty-Ratio-to-Inductor-Current Transfer Functions of DCDC PWM Buck Converter in CCM," IEEE Trans. Ind. Electron., vol. 59, no. 1, pp. 641-643, Jan. 2012.

[5] D. Czarkowski and M. K. Kazimierczuk, "Energy-conservation approach to modeling PWM DC-DC converters," IEEE Trans. Aerosp. and Electron. Syst., vol. 29, no. 3, pp. 1059-1063, July 1993.

[6] Y. Yan, F. C. Lee, and P. Mattavelli, "Analysis and design of average current mode control using a describing-function-based equivalent circuit model," IEEE Trans. Power Electron., vol. 28, no. 10, pp. 4732-4741, Dec. 2012. 